\title{
The crystal structure of RTFDC1 reveals a RING-like pseudoheterodimer responsible for pre-mRNA splicing regulation
}

\author{
Andrew Zhai ${ }^{1}$, Liyan Qiu ${ }^{3}$, Étienne Coyaud ${ }^{3}$, Qun Pan ${ }^{4}$, Benjamin J Blencowe ${ }^{4,5}$ Brian \\ Raught ${ }^{2,3}$, and Gilbert G. Privé ${ }^{1,2,3}$ \\ ${ }^{1}$ Department of Biochemistry, University of Toronto, Toronto, Ontario M5S 1A8, Canada; ${ }^{2}$ Department of \\ Medical Biophysics, University of Toronto, Toronto, ON M5G 1L7, Canada; ${ }^{3}$ Princess Margaret Cancer \\ Centre, University Health Network, 101 College Street, Toronto, ON M5G 1L7, Canada; ${ }^{4}$ Donnelly Centre, \\ University of Toronto, Ontario, M5S 3E1, Canada; ${ }^{5}$ Department of Molecular Genetics, University of \\ Toronto, Ontario, M5S 1A8, Canada
}

The splicing of pre-messenger RNA into mature mRNA by the spliceosome is one of the most dynamic and tightly regulated processes in the eukaryotic cell. While the conjugation of ubiquitin and ubiquitin-like modifiers by RING-type E3 ligases represents a common mechanism for regulating various cellular processes, its involvement in premRNA splicing is not well understood. We have determined the crystal structure of a poorly characterized protein known as Replication Termination Factor Domain Containing 1 (RTFDC1) to a resolution of $2.4 \AA$ A. Surprisingly, while RING-like domains are known to function as homo- or hetero-dimers, the monomeric RTFDC1 crystal structure revealed two RING-like domains assuming a dimer-like formation. Comparison of RTFDC1 to the structure of other RING-like dimers reveals a common dimerization interface. Proteomic analysis of the RTFDC1 interactome through proximity-dependent biotin labeling (BioID) in HEK293 cells revealed an enrichment in components of the spliceosome, particularly those belonging to the U2 snRNP. Finally, to identify a direct effect of RTFDC1 on pre-mRNA splicing, we performed mRNA-seq on RTFDC1 knockdown cells. We observed an effect on mRNA splicing involving predominantly intron retention. These results establish RTFDC1 as a potentially novel E3 ligase regulating pre-mRNA splicing. 